\title{
Synthesis of iron oxide core chitosan nanoparticles in a 3D printed microfluidic device
}

\author{
Mehmet D. Așık (D) • Mesut Kaplan • Barbaros Çetin • \\ Necdet Sağlam
}

Received: 11 October 2020 / Accepted: 19 February 2021 / Published online: 2 March 2021

(C) The Author(s), under exclusive licence to Springer Nature B.V. part of Springer Nature 2021

\begin{abstract}
Nanostructures are capable of major changes in our life. However, the game changing properties of experimental nanostructures mostly are not repeatable for the industry and it is not easy to produce the amount of nanoparticles necessary for the industrial world. Repeatable methods, which do not require highly trained personnel, for industrial-scale production should be developed to transfer the academic research to the use of people. Although there are various successful microfluidics devices that have been designed for
\end{abstract}

M. D. Așık

Department of Medical Biology, Ankara Yıldırım Beyazıt University, Ankara, Turkey

\section{D. Așık $(\varangle) \cdot$ N. Sağlam}

Department of Nanotechnology and Nanomedicine, Hacettepe University, Ankara, Turkey

e-mail: mehmetdoganasik@gmail.com

\section{Kaplan}

Department of Bioengineering, Hacettepe University, Ankara, Turkey

\section{B. Çetin}

Department of Mechanical Engineering, Microfluidics \& Lab-on-a-chip Research Group, Bilkent University, Ankara, Turkey

B. Çetin

UNAM, National Nanotechnology Research Center and Inst. Material Science \& Nanotechnology, Bilkent University, Ankara, Turkey microstructures synthesis, the synthesis of the nanostructures is not an enlightened area and there is a need for research to reach a better state. Especially, the development and design of microfluidics devices for biopolymeric nanoparticles are very important. The biopolymeric nanoparticles have uses in both nanotechnology and nanomedicine especially as theragnostic tools. In this study, a microfluidic device has been modeled, designed, and manufactured for especially iron oxide core chitosan nanoparticles. The microfluidics channels were manufactured by $3 \mathrm{D}$ printing. After nanoparticles synthesized by manufactured device, these particles were characterized, and their properties were examined. In addition to the flow rate, chemical concentrations, and $\mathrm{pH}$, the structure of the microfluidics channel and hurdles have effects on the particle size and particle size distribution. Best results were obtained with $120-120 \mathrm{ml} / \mathrm{h}$ flow rates and 0.06 $0.03 \%$ concentrations at $\mathrm{pH} 4.5$ for chitosantripolyphosphate couple. The nanoparticles that were produced in microchannels with hurdles under these conditions have a DLS measurement of $190 \pm 15 \mathrm{~nm}$ in diameter with $69 \%$ intensity. In conclusion, the 3D printed microfluidic channels are able to synthesize nanoparticles in a reproducible way with or without iron oxide core.

Keywords Microfluidics · Iron oxide nanoparticles · Chitosan nanoparticles $\cdot$ Microfluidic nanoparticle synthesis $\cdot$ Nanofabrication 


\section{Introduction}

The term nanoparticle is used for nano-size crystals, rods, stars, and many shapes, but is mainly preferred for non-hollow spherical nanostructures. Although the theoretical foundations were laid out in the 1960s, the emergence of the first nanoparticles dates back to the 1990s. After this date, nanoparticle studies, which gained great momentum with many researchers, enabled the production of a wide variety of nanoparticles, both metal, polymer, and biological. In a 1996 review, it was proposed that micro-emulsion can be used to produce polymeric nanoparticles as well as metallic, semiconductor, superconductive, and magnetic particles (Eastoe and Warne 1996). In 1998, it was stated in the European Research Agency's assessment report that synthesizing nanoparticles in the gas phase had been possible (Fissan and Schoonman 1998). However, the studies carried out in these years are the basic studies conducted without considering the industrial applications needs. Subsequent studies have sought ways to produce high volumes of nanoparticles. A study conducted in 2003 claims that by pyrosynthesis, also known as flame spray synthesis, up to $1.1 \mathrm{~kg}$ of nanoparticles can be produced per hour (Mueller et al. 2003). Although some studies gave good results for metals such as zirconium, aluminum, and inorganic materials such as silica, the application of this method with polymeric and biological materials is still a problem.

Polymeric nanoparticles are suitable candidates for drug transport and in this sense will provide new developments in the field of medicine (Schaffazick et al. 2003). These nanoparticles, which are unique due to their electronic, photonic, catalytic properties, will again produce unique biomaterials in terms of the properties and functions of their integration with biomaterials (Katz and Willner 2004). The studies established in the following years mention the necessity of another feature of polymeric nanoparticles to be used in the body. In a 2007 review, it was mentioned that the focus of the current studies was on the development of biocompatible nanoparticles which could be targeted to cancerspecific markers, and it was shown that a number of clinical and preclinical investigations had been conducted for the diagnosis and treatment of cancer using imaging and treatment agents (Pridgen et al. 2007).

Biopolymeric nanoparticles have been the subject of many researches, especially in the last decade. Studies on the use of biopolymeric nanoparticles in sectors such as medicine, pharmacy, food, and animal husbandry have been pioneering in this field (Jones and McClements 2011). Since biopolymeric nanoparticles are biocompatible and/or biodegradable, they are preferred in many fields which include controlled drug/ gene transport, tissue engineering, and imaging of specific parts of the DNA structure. Cancer, diabetes, allergy, infection, and inflammation are the most successful topics in the use of these nanoparticles for therapeutic purposes (Nitta and Numata 2013). For instance, for the treatment of diabetes, polymers such as chitosan, alginate, and dextran sulfate are used for oral administration of insulin (Lopes et al. 2016). Similarly, the phenomenon of enhanced permeability and retention (EPR) makes biopolymeric nanoparticles an important tool for cancer treatment (Prabhakar et al. 2013). Moreover, biopolymeric nanoparticles, which are also an important element not only for drug delivery but also for genetic therapies, by encapsulating siRNA and miRNAs, enables their use in cancer treatment (Nitta and Numata 2013). Chitosan nanoparticles are one of the most studied biopolymeric nanoparticles. Another area that biopolymeric nanoparticles are used is diagnosis. In particular, biopolymeric nanoparticles containing iron or various contrast agents are used to enhance the success of the techniques currently used for diagnosis. There are many biopolymeric nanoparticles developed for techniques such as computed tomography, ultrasound, and magnetic resonance imaging. In a review published in 2014, it was emphasized that the studies in this field have increased in the previous decade (Cormode et al. 2014). There is a common acceptance that the biopolymeric nanoparticles including metal nanostructures have a great potential for using as theragnostic agents.

For the synthesis of biopolymeric nanoparticles, generally, liquid phase methods are used. Emulsification, double emulsion, suspension polymerization, micelle formation, solvent substitution, salt precipitation, sol gel, and ionic gelation methods are the main technics used. The use of these methods varies according to the structural and chemical properties of the biological molecule for the nanostructure production. For example, liposomes are obtained from oils with polar and apolar ends by micelle formation. Another method is the double emulsion method for the production of nanocapsules. The easiest and most well-known of these methods is undoubtedly the ionic gelation method (Skorik et al. 2017; Mukhopadhyay et al. 2013). For 
instance, chitosan molecule has protonated amine groups that interact with the negative phosphate groups on tripolyphosphate and this reaction is $\mathrm{pH}$ dependent because degree of ionization of TPP and chitosan is dependent to different $\mathrm{p} K_{\mathrm{a}}$ values (Pati et al. 2011). On the contrary of being easy, it is very difficult to form nanostructures in the desired size distribution for biopolymeric nanoparticles. The reason for this is that the monitoring and control of the parameters affecting the formation of nanostructures in discrete systems are not possible. Differences in micro- or nano-dimensions in temperature, $\mathrm{pH}$, concentration, quantity, and volumes cause major changes in nanoparticles (Calvo et al. 1997; Kheiri et al. 2017). In addition, it is not possible to control changes based on personal differences and the need for trained personnel. For this reason, the production methods for polymeric and biopolymeric nanoparticles are not sufficient for industrial applications and the idea of developing a tunable reactor that will produce repeatable nanoparticles has emerged. In particular, Shchukin et al., who conducted a reactor study for the production of organic nanoparticles, suggested that the nanomaterials to be synthesized in a micrometer cube volume has the possibility of (1) having high catalytic properties due to the surface morphology and high surface area of the nanoparticle, (2) having high stability against to aggregation, (3) having high purity due to protected reactors, (4) being open to modifications, and (5) having hierarchical architecture with multi-step synthesis (Shchukin and Sukhorukov 2004). The approach that microfluidic dynamics-based systems could be used for nanoparticle synthesis has been proposed and studies showing that nanoparticles can be synthesized with great sensitivity and control within microchannels have begun to emerge (Marre and Jensen 2010). These systems, also known as nanoparticle microreactors, enabled nanoparticle synthesis by many different methods. Zhao's review in 2011 revealed that nanoparticles can be produced by (1) 2-D mixing-based, (2) gas-liquid section, (3) droplet-based, and (4) coalescence-based systems; however, contrary to these structures, which are the application of different derivatives for inorganic particles, it is stated that there were not enough studies that have been done for organic and polymeric structures (Zhao et al. 2011). On the other hand, a research group focused on this field and with their studies they developed a system for the production of polymeric nanoparticles (Karnik et al. 2008). The nanoparticles obtained in these studies are expected to produce nanoparticles with a higher yield and a narrower distribution in terms of size than a batch system. Indeed, a study published in 2016 comparing the amounts of polymeric nanoparticle production showed that it is possible to produce $288 \mathrm{mg}$ of particles per hour, thus producing from 1 to $7 \mathrm{~g}$ of polymeric nanoparticles per day (Baby et al. 2017). In recent years, many studies focused on microfluidics nanoparticle synthesis and solve the problems of these systems (Luo et al. 2019; Lu et al. 2016; Kibar et al. 2019). Many researchers tried different variables such as flow rate to increase the monodispersity of particles (Abstiens and Goepferich 2019; Bressan et al. 2019; Zhao et al. 2019). Furthermore, two staged microfluidics systems were developed for more complex synthesis (Ma et al. 2020). Also, production of silver loaded polymer particles became possible with late studies (Yang et al. 2016). Although many efforts focused on this area, daily life experience underlines standing industrial and academic problems such as SARS-cov2 vaccination production. Most of the SARS-Cov2 vaccines are based on lipid nanoparticles and there is a production range that limits the vaccination procedures which causes increased mortality, and microfluidic platforms can be used for mass production of the nanoparticles to overcome this problem (No author 2020; Tang et al. 2020). The mass production of lipid/polymeric particles is possible with low polydispersity index (Colmenares Roldán et al. 2018; Kim et al. 2012). Moreover, the lipid vesicle synthesis and siRNA gene therapy nanoparticles can be produced by high throughput turbulent jet devices (Lim et al. 2014). On the other hand, a review in 2015 highlights several of the methods for the encapsulation that have specific relevance to industrial formulations and the challenges in the literature. These challenges include robust stability, high production capacity without losing complexity and maintain the performance of the formulation with an acceptable cost profile (Andrade et al. 2015). Recent studies focus on multistage microfluidics systems to solve complexity problem (Volk et al. 2020).

The aim of this study is to develop a microfluidic dynamics-based system, in which polymeric and biopolymeric nanoparticles can be produced in high quantities especially for theragnostic applications. The characterization of the nanoparticles obtained from this system and determination of the best manufacturing methods by defining the superiority of them against each other, is an incontrovertible necessity for industrial nano age in the future. 


\section{Experimental design}

The designed microfluidics device (Fig. 1a) including microchannels in Fig. 1c with hurdles in Fig. 1d was produced (Fig. 1b) by using a 3D printer (ProJet 3500 HD Max). ABS like material (VisiJet M3) and supporting wax were used for production. After printing, isopropyl alcohol and water solution was applied to the microchannels to remove the supporting wax at $50{ }^{\circ} \mathrm{C}$ with sonication. Following to 1-h sonication, channels were left in isopropyl alcohol and water solution for $24 \mathrm{~h}$.

Nanoparticle production in microfluidics channel

Nanoparticle production was performed by injecting the chitosan and sodium tripolyphosphate (TPP) solutions from inlet 1 and inlet 2 and nanoparticles were collected from outlet (Sigma Aldrich, USA, 448869, 72061). Chitosan was dissolved in $1 \%$ acetic acid and TPP was dissolved in distilled water according to the different concentrations in Tables 3 and 4. Injectors $(50 \mathrm{~mL})$ were filled with chitosan and TPP solutions. The injectors were placed to injection pumps and tubing was placed between injectors and microfluidics device (Supplemental figure 1). Injection pumps were used to inject solutions at a constant rate. Following the synthesis, collected nanoparticles were washed 3 times with centrifuge at $21000 \times g$ with $1.5 \mathrm{~mL}$ centrifuge tubes (Hettich Universal 320R, Germany). Variables including volumetric flow rate, $\mathrm{pH}$, formulation, and TPP and chitosan concentrations were tested to observe their effects on nanoparticle formation $(n=3)$.

Volumetric flow rate and $\mathrm{pH}$ experiments

Volumetric flow rate pairs were selected between 120 and $40 \mathrm{ml} / \mathrm{h}$. \%0.06 chitosan in $1 \%$ acetic acid and $\% 0.03$ TPP solutions were prepared. Later, $\mathrm{pH}$ of the chitosan arranged to the 4.5 with $0.1 \mathrm{M} \mathrm{NaOH}$ and different flow rate pairs were tested (Table 1). For the $\mathrm{pH}$ experiments, \%0.06 chitosan in $1 \%$ acetic acid and \%0.03 TPP solutions were prepared. Later, different chitosan $\mathrm{pH}$ values which were arranged with $0.1 \mathrm{M}$ $\mathrm{NaOH}$ were tested (Table 2).

\section{Concentration experiments}

Concentration experiments include different chitosan and TPP concentrations. With $120 \mathrm{ml} / \mathrm{h}$ volumetric flow rate pair and at $\mathrm{pH}=4.5$, the formulations in Tables 3 and 4 were tested.
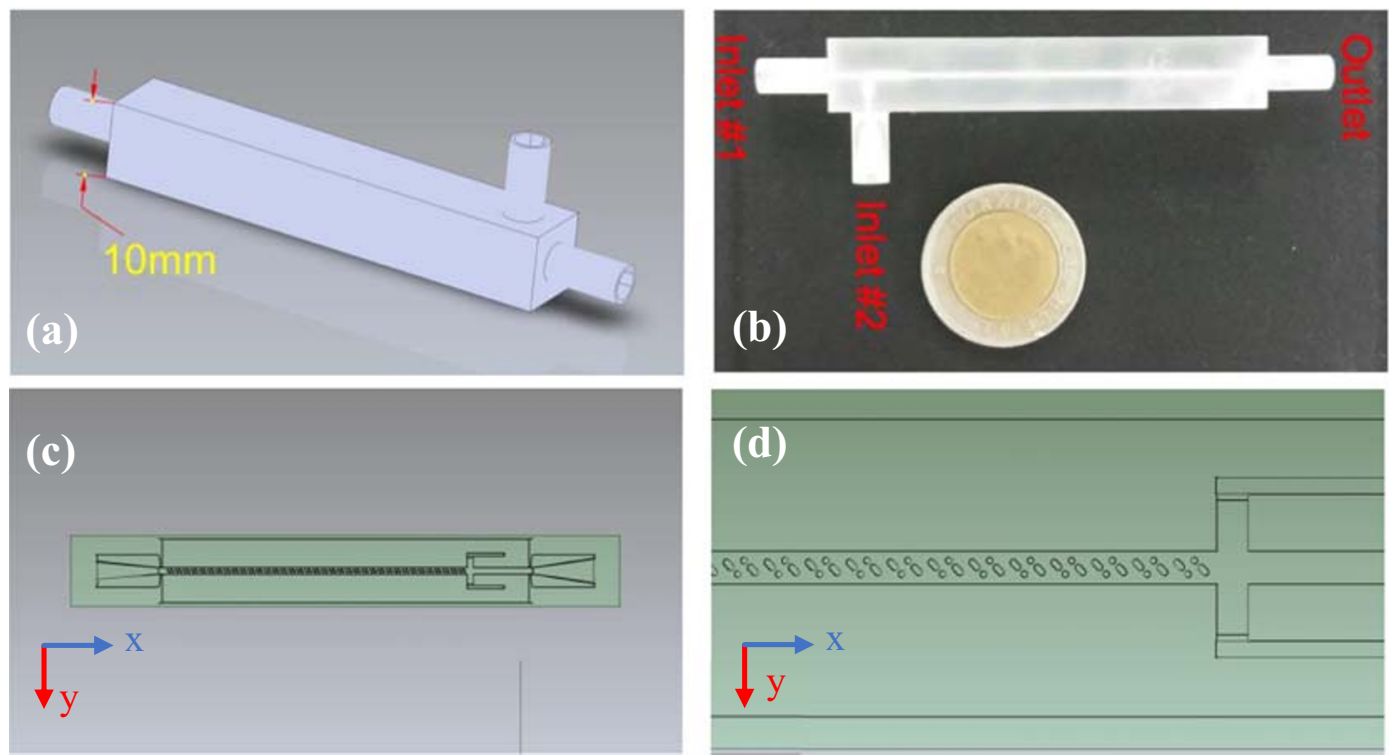

Fig. 1 Microfluidics channels with hurdles (a) 3d solid model of the microfluidics device, (b) $3 \mathrm{~d}$ printed microfluidics device, (c) microfluidics channel, and (d) hurdles in the microfluidics channel 
Table 1 Volumetric flow rate experiments

\begin{tabular}{lll}
\hline & Chitosan [ml/hour] & TPP $[\mathrm{ml} /$ hour $]$ \\
\hline Experiment 1 & 120 & 40 \\
Experiment 2 & 120 & 80 \\
Experiment 3 & 120 & 120 \\
Experiment 4 & 80 & 120 \\
Experiment 5 & 40 & 120 \\
\hline
\end{tabular}

Synthesis of iron oxide core chitosan nanoparticles

For the synthesis of iron oxide core chitosan nanoparticles, the coprecipitation technique was applied by precipitating $\mathrm{Fe}+2$ and $\mathrm{Fe}+3$ with $\mathrm{NaOH}$. Following to wash steps, iron oxide nanoparticles dispersed in chitosan at $\mathrm{pH} 4.5$ and injected to the microchannel with a flow rate of $120 \mathrm{ml} / \mathrm{h}$. Collected nanoparticles were washed with a centrifuge at $21,000 \times g 3$ times.

\section{Characterization of nanoparticles}

Nanoparticles were characterized with dynamic light scattering (DLS) for particle size (Malvern, USA). Nanoparticles dispersed in water and placed in DLS for particle size measurements. A scanning electron microscope (SEM) (Quanta 200 FEG, FEI, USA) and transmission electron microscopy (TEM) (Tecnai G2 F30, Atlanta, USA) was employed for morphology characterization. For the preparation of the TEM specimen, a small amount of the nanoparticle was dispersed in a few milliliters of water and the suspension were placed onto a carbon coated grid (Saleh and Gupta 2012). Energy dispersive X-ray spectroscope (EDX) was used for element analysis of the samples (Saleh 2015). Additionally, FTIR is used to analyze the bonds in the chitosan and iron oxide chitosan nanoparticles.

Table 2 pH Experiments

\begin{tabular}{ll}
\hline & $\mathrm{pH}$ of chitosan \\
\hline Experiment 1 & 4.2 \\
Experiment 2 & 4.4 \\
Experiment 3 & 4.5 \\
Experiment 4 & 4.6 \\
Experiment 5 & 4.8 \\
\hline
\end{tabular}

Table $30.3 \%$ chitosan and different TPP concentrations experiments

\begin{tabular}{lll}
\hline $\mathrm{W} / \mathrm{V}$ & Chitosan [\%] & TPP [\%] \\
\hline Experiment A1 & 0.300 & 0.200 \\
Experiment A2 & 0.300 & 0.100 \\
Experiment A3 & 0.300 & 0.075 \\
Experiment A4 & 0.300 & 0.050 \\
Experiment A5 & 0.300 & 0.025 \\
\hline
\end{tabular}

\section{Results}

Volumetric flow rates critically affected nanoparticle size. Figure 2 shows that straight microchannels and microchannels with hurdles have different results for 5 different volumetric flow rate pairs. In Fig. 2a, particles produced in straight microchannels were larger than the DLS detection limit. On the other hand, nanoparticles that were produced in microchannels with hurdles have a hydrodynamic diameter of $295 \mathrm{~nm}$ with $100 \%$ intensity. In Fig. 2b, straight microchannels resulted in $825 \mathrm{~nm}$ particles with $100 \%$ intensity and microchannels with hurdles resulted in $342 \pm 50 \mathrm{~nm}$ particles with $77 \%$ intensity. In Fig. 2c, the nanoparticles that are produced in straight microchannels had a peak at $164 \mathrm{~nm}$ with $14 \%$ intensity and the average diameter was $364 \pm 269 \mathrm{~nm}$. The nanoparticles that were produced in microchannels with hurdles have a diameter of $190 \pm 15 \mathrm{~nm}$ with $69 \%$ intensity. The experiment results in Fig. 2d and e are similar. Both graphs have 2 peak values with similar intensities. The nanoparticles in the straight microchannels with the highest peaks had diameters of $255 \mathrm{~nm}$ and $295 \mathrm{~nm}$ respectively, and the nanoparticles in microchannels with hurdles had diameters of $825 \mathrm{~nm}$ and $295 \mathrm{~nm}$ respectively.

$\mathrm{pH}=4.2, \mathrm{pH}=4.4$, and $\mathrm{pH} 4.6$ experiments gave similar size and size distribution results for both microchannels. Although $\mathrm{pH}=4.5$ and $\mathrm{pH} 4.8$ had sim-

Table $4 \quad 0.06 \%$ chitosan and different TPP concentrations experiments

\begin{tabular}{lll}
\hline W/V & Chitosan [\%] & TPP [\%] \\
\hline Experiment B1 & 0.06 & 0.08 \\
Experiment B2 & 0.06 & 0.04 \\
Experiment B3 & 0.06 & 0.03 \\
Experiment B4 & 0.06 & 0.02 \\
Experiment B5 & 0.06 & 0.01 \\
\hline
\end{tabular}




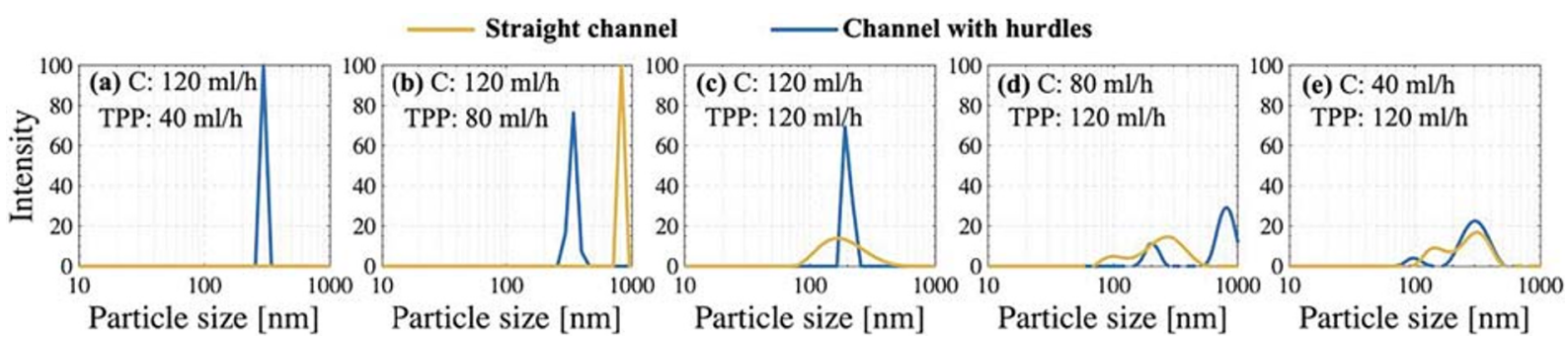

Fig. 2 DLS results of volumetric flow rate experiments for B3 formulation: (a) Chi: $120 \mathrm{ml} / \mathrm{h}$ - TPP: 40ml/h, (b) Chi: $120 \mathrm{ml} / \mathrm{h}$ - TPP: 80ml/h, (c) Chi: $120 \mathrm{ml} / \mathrm{h}$ —TPP: 120ml/h, (d) Chi: $80 \mathrm{ml} / \mathrm{h}$-TPP: $120 \mathrm{ml} / \mathrm{h}$, (e) Chi: $40 \mathrm{ml} / \mathrm{h}$-TPP: $120 \mathrm{ml} / \mathrm{h}$

ilar size distributions to other $\mathrm{pH}$ experiments, sharper peaks were observed for the microchannels with hurdles (Fig. 3).

When concentration experiments evaluated, there is a drastic change in particle size and size distribution. From formulation A1 to A5, the effect of decreasing TPP can be seen in Fig. 4. While TPP concentration was decreasing, the sharpness of the peak increased which indicates a better size distribution. However, the particle size was also increased which is not a desirable consequence. A similar result was also observed for B1 to B3. B4 and B5 formulations did not yield enough nanoparticles to measure.

The FT-IR spectrum of chitosan shows the characteristic peak in the region $3279-3289 \mathrm{~cm}^{-1}$ which corresponds to N-H and O-H stretching, as well as intramolecular hydrogen bonds. The peak at $1637 \mathrm{~cm}^{-1}$ belongs to $\mathrm{C}=0$ stretching of amide $\mathrm{I}$ and the peak at $1338 \mathrm{~cm}^{-1}$ belongs to C-N stretching of amide III. The presence of residual $\mathrm{N}$-acetyl groups can be confirmed by the bands at around $1637 \mathrm{~cm}^{-1}$ and $1338 \mathrm{~cm}^{-1}$ (Fernandes Queiroz et al. 2015). We can observe a peak at $1549 \mathrm{~cm}^{-1}$ which shows the presence of N-H bending of amide II. The $\mathrm{CH}_{2}$ bending shows a peak at $1406 \mathrm{~cm}^{-1}$. The band at 1102 and $1020 \mathrm{~cm}^{-1}$ corresponds to $\mathrm{C}-\mathrm{O}$ stretching. The absorption band at $1150 \mathrm{~cm}^{-1}$ belongs to asymmetric stretching of the C-O-C bridge. The signal at $896 \mathrm{~cm}^{-1}$ can be interpreted as the $\mathrm{CH}$ bending out of the plane
(Fernandes Queiroz et al. 2015). The FTIR spectrum of magnetite shows strong absorption band which is located at around $554 \mathrm{~cm}^{-1}$. This band corresponds to the Fe$\mathrm{O}$ stretching mode of tetrahedral and octahedral sites (the band at $554 \mathrm{~cm}^{-1}$ ). There is strong stretching absorption band at $554 \mathrm{~cm}^{-1}$ which belongs to $\mathrm{Fe}-\mathrm{O}$ bond. According to Stoia, the band at $554 \mathrm{~cm}^{-1}$ corresponds the magnetite since the band located at $554 \mathrm{~cm}^{-1}$ is sharp and symmetric and only a weak shoulder around $451 \mathrm{~cm}^{-1}$ exists (Stoia et al. 2016). The intense band at $554 \mathrm{~cm}^{-1}$ can be assigned to IR active $T_{1 \mathrm{u}}$ mode which corresponds to the vibration of the $\mathrm{Fe}^{2+}-\mathrm{O}^{2-}$ functional group (Khalil 2015). The band between 1651 and 1663 $\mathrm{cm}^{-1}$ shows $\mathrm{NH}_{2}$ group in the compound. The peak at $1432 \mathrm{~cm}^{-1}$ can be interpreted as $\mathrm{O}-\mathrm{H}$ bending which belongs to primary amine. The FTIR spectrum shows water molecule stretching vibration band at around 3250 $\mathrm{cm}^{-1}$ (Stoia et al. 2016) (Fig. 5).

In Fig. 6a, SEM images of B3 formulation nanoparticles produced in the straight channels have a size distribution of 100 to $600 \mathrm{~nm}$. Moreover, there are aggregated structures and particles that are not in fully spherical shape. In Fig. 6b, the B3 formulation nanoparticles produced in microchannels with hurdles can be observed. The size of the particles varies between 100 and $300 \mathrm{~nm}$ in a spherical shape.

In Fig. 7a and b, SEM images of iron oxide core chitosan nanoparticles produced in the straight

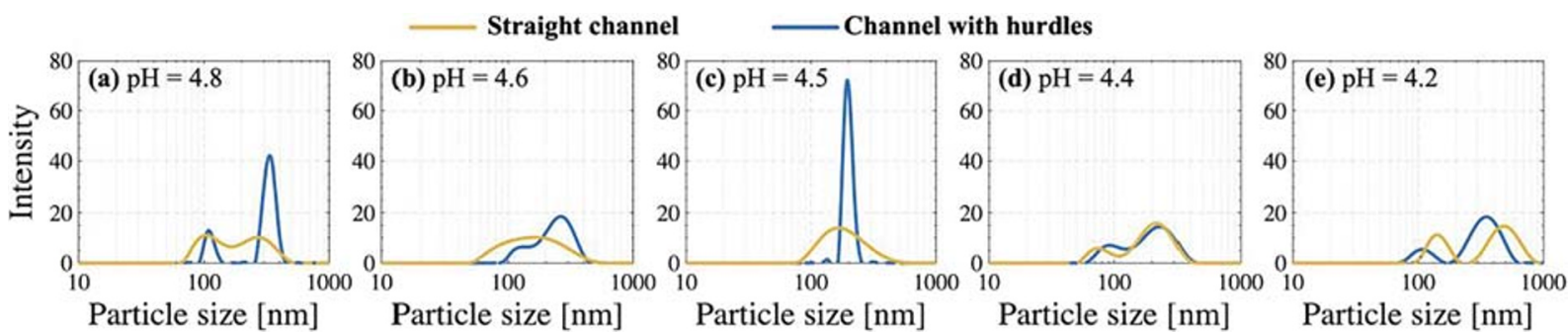

Fig. 3 DLS results of $\mathrm{pH}$ experiments for B3 formulation and Chi: $120 \mathrm{ml} / \mathrm{h}-\mathrm{TPP}: 120 \mathrm{ml} / \mathrm{h}$ flow conditions: $(\mathbf{a}) \mathrm{pH}=4.8,(\mathbf{b}) \mathrm{pH}=4.6,(\mathbf{c})$ $\mathrm{pH}=4.5$, (d) $\mathrm{pH}=4.4$, (e) $\mathrm{pH}=4.2$ 

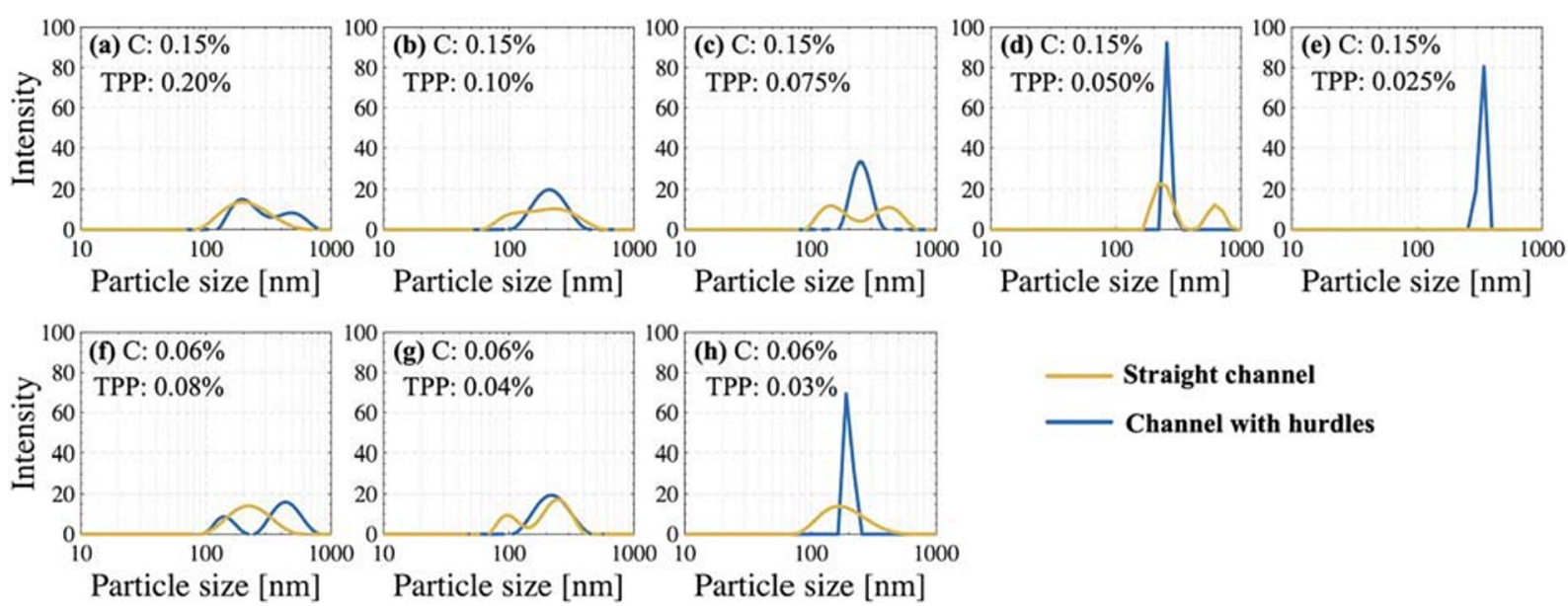

Fig. 4 DLS results of concentration and formulation experiments for $\mathrm{pH}=4.5$ and Chi: $120 \mathrm{ml} / \mathrm{h}-\mathrm{TPP}: 120 \mathrm{ml} / \mathrm{h}$. Formulations (a) A1, (b) A2, (c) A3, (d) A4, (e) A5, (f) B1, (g) B2, (h) B3

channel and the microchannels with hurdles can be seen. In Fig. 7a, iron oxide core chitosan nanoparticle size varies between 30 and $200 \mathrm{~nm}$. However, the smaller particles lack proper chitosan coating. They have a more angular structure than spherical. On the other hand, nanoparticles in Fig. $7 \mathrm{~b}$ have spherical shapes and their size varies between 60 and $100 \mathrm{~nm}$ which indicates a better and consistent coating of all nanoparticles.
In Fig. 8, TEM images of iron oxide, chitosan, and iron oxide core chitosan nanoparticles can be seen. The iron oxide nanoparticles were monodisperse and had a size distribution between 20 and $40 \mathrm{~nm}$. The TEM image of chitosan nanoparticles has a cloudy image of $110 \mathrm{~nm}$. On the other hand, iron oxide core chitosan nanoparticle has an intense core with a cloudy image outside (Fig. 8b). Moreover, the iron oxide and iron oxide core
Fig. 5 FTIR spectrums of (a) chitosan nanoparticles and (b) iron oxide core chitosan nanoparticles

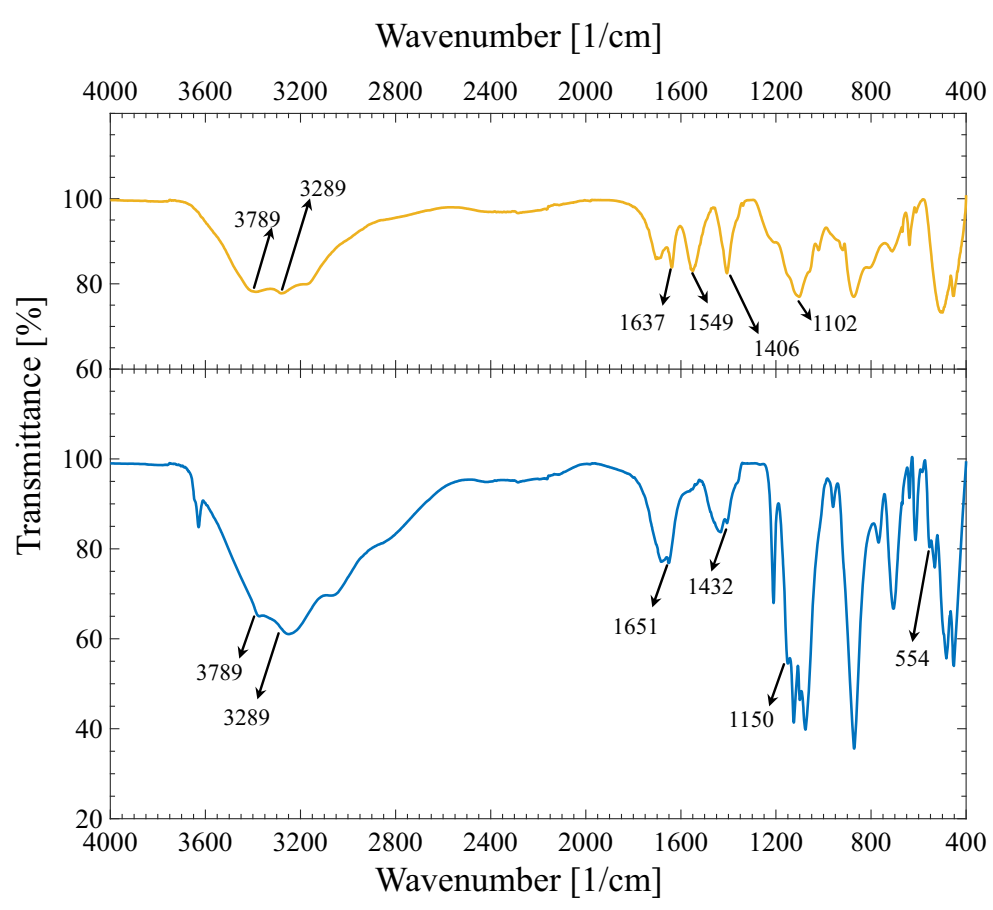



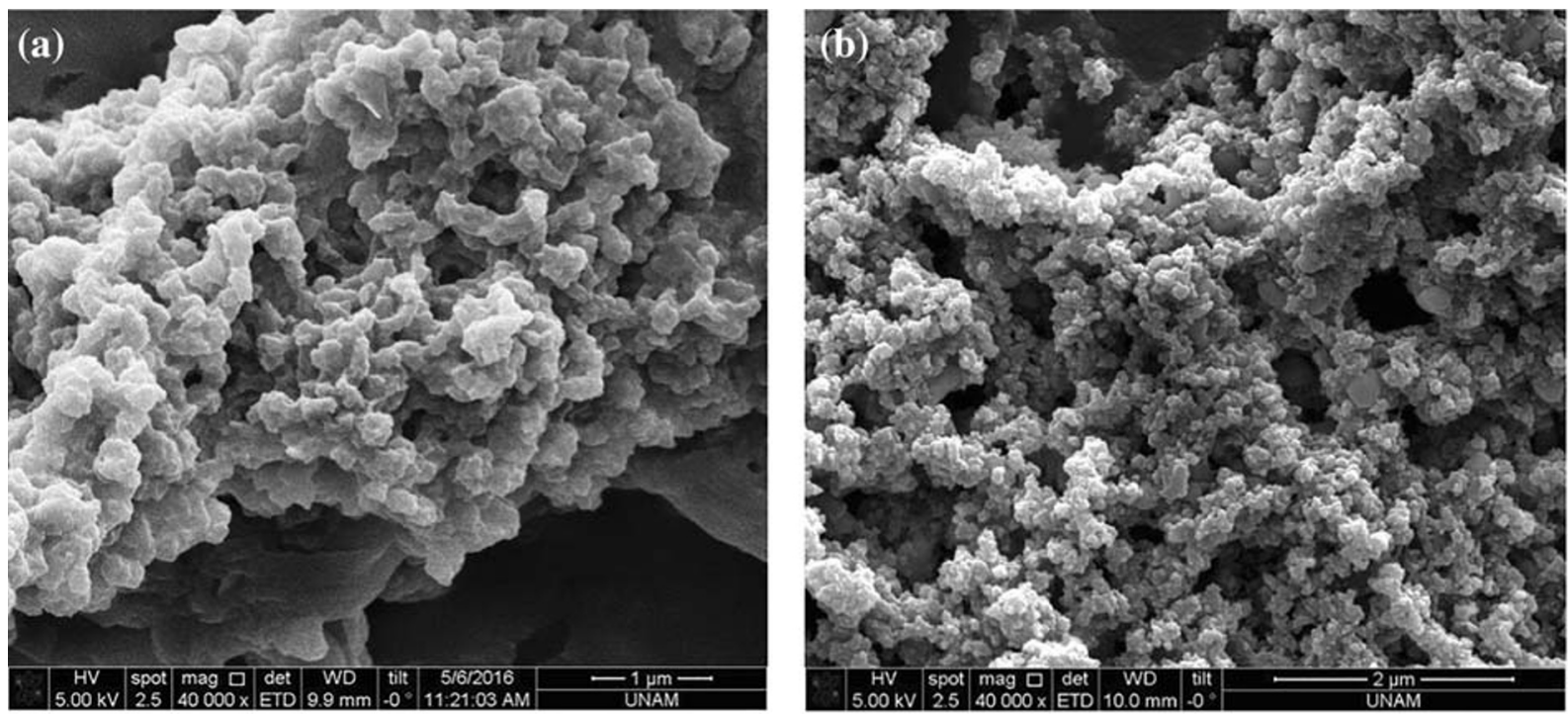

Fig. 6 SEM images of B3 formulation in (a) straight channel and (b) microchannels with hurdles

chitosan nanoparticles were affected with a magnetic field which can be observed in Fig. 8d.

\section{Discussion}

In this study, results include the effect of microfluidics design, volumetric flow, $\mathrm{pH}$, and concentration and different formulations. Previous studies indicate that the mixing of chitosan and TPP can be modeled as a function of channel geometry/shape and channel length. However, it is possible to avoid aggregate formation and achieve optimum mixing by adding obstacles to the channel design. The geometric design was chosen as a $7-\mathrm{cm}$ channel with a flow focusing junction model. The results were less aggregated compared to the previous design in 2014 (Cetin et al. 2014). However, both systems have a similar problem. The concentrations that are used in batch systems cause clogs in the entrance of the channel due to the gelation. Other researchers observed similar results and they indicated that adding another fluid into the system may create another diffusion surface and may solve the clogging (Pessoa et al. 2017). In our study, the lower concentrations give a similar
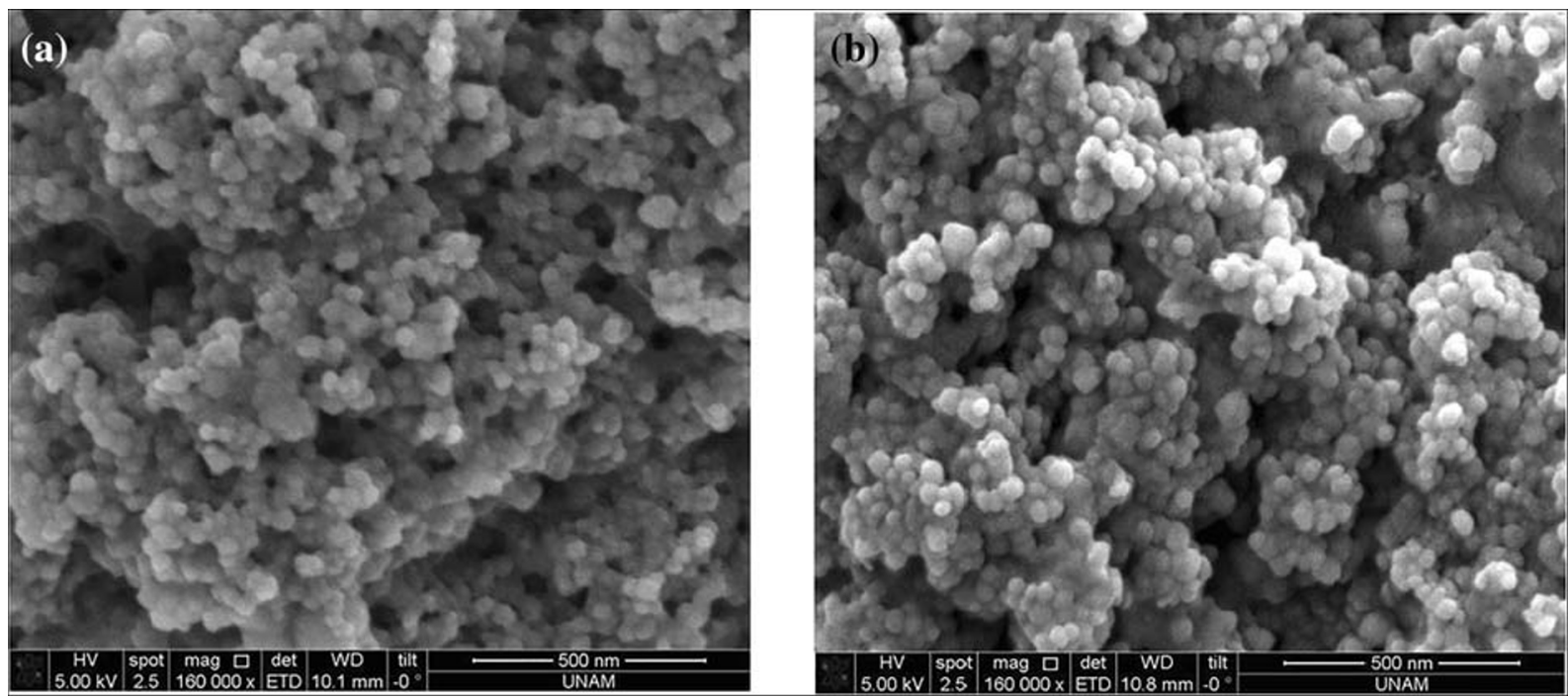

Fig. 7 SEM images of iron oxide core chitosan nanoparticles from the (a) straight channel and (b) the microchannels with hurdles 

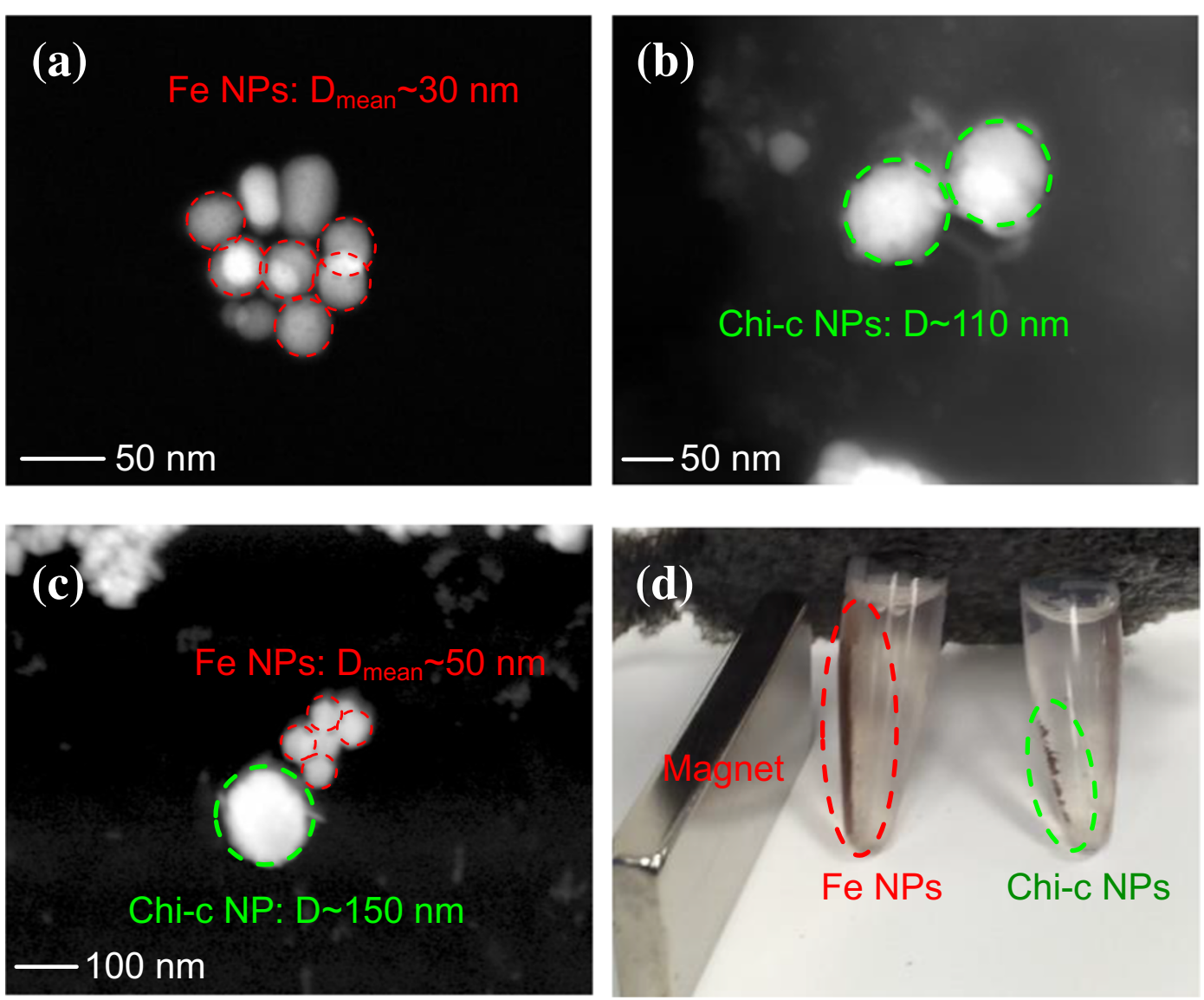

Fig. 8 TEM images of (a) iron oxide nanoparticles, (b) chitosan nanoparticles, (c) iron oxide core chitosan nanoparticles from the (d) magnetized iron oxide and iron oxide core chitosan nanoparticles

outcome without adding another fluid to the system. In concentration experiments, formulation B3 gave the best and most repeatable results. Although, B4 and B5 formulations gave good results, they drop behind due to low yielding. Like the batch system, $\mathrm{pH}$ differences affect the particle size and formation and in B3 formulation, the effect of the channels with obstacles can be observed best with $\mathrm{pH}$ 4.5. Both formulation and volumetric flow experiments indicate a decrease of TPP amount compared to chitosan amount results in better particle size distribution. These results approve the mixing would increase as in many simulation models. On the other hand, increased retention time may also cause clogging and aggregates. For this reason, the volumetric flow rate pair $120 \mathrm{ml} / \mathrm{h}-120 \mathrm{ml} / \mathrm{h}$ probably last longer with optimum results without clogging or causing nanoparticle aggregations.

The SEM and TEM results indicate that there are morphological differences between the nanoparticles produced by straight microchannel and microchannel with the hurdles. The straight channels produce particles with larger size distribution compared to the channels with hurdles. Moreover, the sphericity of the nanoparticles also differs from straight channel compared to the channel with the hurdles. Furthermore, the iron oxide core chitosan nanoparticles that were produced in the channels with hurdles are rounder and less angular. SEM images indicate a better chitosan coating on iron oxide nanoparticles. This is another proof of that the chitosan and TPP solutions mixes better in the channel with the hurdles. In addition, elemental analysis results prove that iron oxide cores were covered with chitosan to form iron oxide core chitosan nanoparticles (see Figures S1, S2 and S3 in the Supplementary file). Carbon and oxygen peaks increased when the electron beam focused on the chitosan layer. Moreover, EDX graph also indicates that there is more carbon in the iron oxide core chitosan nanoparticles compared to the just iron oxide particles. 
It is possible to use multistage microfluidic platforms for complex chemical reactions (Volk et al. 2020). Therefore, large scale production of nanoparticles may be accomplished by parallel use of multiple reactors. 3D printing is a tool to develop and produce certain devices and it is also very useful for producing of tens of $3 \mathrm{D}$ reactors. However, if 100 s or 1000 s are needed, simpler multistage designs can be applied to the devices and these may be manufactured by using gold standard plastic injection technique.

By applying aforementioned flow rates, it is possible to get $240 \mathrm{ml}$ of nanoparticle solution and with a chip that includes 5 microchannels and 40 chips it is possible to reach 168 grams of nanoparticles. This is about $4 \mathrm{~kg}$ of biopolymeric nanoparticles per day. It would be possible to fine-tune the size and size distribution of the nanoparticles by adjusting the $\mathrm{pH}$ and volumetric flow rates. Further studies should include an electronic control system for pumps to develop an industrialized automated system to synthesize magnetic chitosan nanoparticles.

In conclusion, the concentration of the chitosan and TPP, $\mathrm{pH}$ of the chitosan, and the amount of the chemicals/flow rates during the synthesis and channel structure are all very important for the resulting particles. These values are optimized for the best particle size and particle size distribution for the chitosan particles, especially iron oxide core chitosan particles. However, each different nanoparticle synthesis in microfluidics with different materials need a lot of optimization study before commercialized and scaled-up production. Although microfluidics systems might reduce the need for technical knowledge to a certain level, nanoparticle synthesis in industrial scale will continue to be a huge problem for a while.

Supplementary Information The online version contains supplementary material available at https://doi.org/10.1007/s11051021-05171-y.

Acknowledgements We thank Duygu Torun and Daniel Aaron Press for the critical reading of manuscript.

Author contribution Conceptualization: N.S., M.A., and B.Ç.; methodology: M.A., M.K, and B.Ç.; investigation: M.A, M.K.; writing - original draft preparation: M.A.; review and editing: and B.C..; All authors have read and agreed to the published version of the manuscript.

\section{Compliance with ethical standards}

Conflict of interest The authors declare no conflict of interest.

\section{References}

Abstiens K, Goepferich AM (2019) Microfluidic manufacturing improves polydispersity of multicomponent polymeric nanoparticles. J Drug Deliv Sci Technol 49:433-439

Andrade B, Song Z, Li J, Zimmerman SC, Cheng J, Moore JS, Harris K, Katz JS (2015) New frontiers for encapsulation in the chemical industry. ACS Appl Mater Interfaces 7(12): 6359-6368. https://doi.org/10.1021/acsami.5b00484

Baby T, Liu Y, Middelberg APJ, Zhao C-X (2017) Fundamental studies on throughput capacities of hydrodynamic flowfocusing microfluidics for producing monodisperse polymer nanoparticles. Chem Eng Sci. https://doi.org/10.1016/j. ces.2017.04.046

Bressan LP, Robles-Najar J, Adamo CB, Quero RF, Costa BM, de Jesus DP, da JAF S (2019) 3D-printed microfluidic device for the synthesis of silver and gold nanoparticles. Microchem J 146:1083-1089

Calvo P, Remuñan-López C, Vila-Jato JL, Alonso MJ (1997) Chitosan and chitosan/ethylene oxide-propylene oxide block copolymer nanoparticles as novel carriers for proteins and vaccines. Pharm Res 14(10):1431-1436. https://doi. org/10.1023/A:1012128907225

Cetin B, Asik MD, Taze S (2014) Design and fabrication of a microfluidic device for synthesis of chitosan nanoparticles. J Nanotechnol Eng Med 4(3):031004-031006. https://doi. org/10.1115/1.4026287

Colmenares Roldán GJ, Agudelo Gomez LM, Carlos Cornelio JA, Rodriguez LF, Pinal R, Hoyos Palacio LM (2018) Production of polycaprolactone nanoparticles with low polydispersity index in a tubular recirculating system by using a multifactorial design of experiments. J Nanopart Res 20(3): 68. https://doi.org/10.1007/s11051-018-4168-8

Cormode DP, Naha PC, Fayad ZA (2014) Nanoparticle contrast agents for computed tomography: a focus on micelles. Contrast Media Mol Imaging 9(1):37-52. https://doi. org/10.1002/cmmi.1551

Eastoe J, Warne B (1996) Nanoparticle and polymer synthesis in microemulsions. Curr Opin Colloid Interface Sci 1(6):800 805

Fernandes Queiroz M, Melo KRT, Sabry DA, Sassaki GL, Rocha HAO (2015) Does the use of chitosan contribute to oxalate kidney stone formation? Marine Drugs 13(1):141-158

Fissan HJ, Schoonman J (1998) Vapor-phase synthesis and processing of nanoparticle materials (NANO) - A European science foundation (ESF) program. J Aerosol Sci 29(5-6): 755-757. https://doi.org/10.1016/s0021-8502(98)00773-3

Jones OG, McClements DJ (2011) Recent progress in biopolymer nanoparticle and microparticle formation by heat-treating electrostatic protein-polysaccharide complexes. Adv Colloid Interf Sci 167(1-2):49-62. https://doi.org/10.1016/j. cis.2010.10.006

Karnik R, Gu F, Basto P, Cannizzaro C, Dean L, Kyei-Manu W, Langer R, Farokhzad OC (2008) Microfluidic platform for controlled synthesis of polymeric nanoparticles. Nano Lett 8(9):2906-2912. https://doi.org/10.1021/nl801736q

Katz E, Willner I (2004) Integrated nanoparticle-biomolecule hybrid systems: synthesis, properties, and applications. Angew Chem-Int Ed 43(45):6042-6108. https://doi.org/10.1002 /anie. 200400651 
Khalil M (2015) Co-precipitation in aqueous solution synthesis of magnetite nanoparticles using iron (III) salts as precursors. Arab J Chem 8:279-284. Elsevier. https://doi.org/10.1016/J.ARABJC

Kheiri A, Moosawi Jorf SA, Malihipour A, Saremi H, Nikkhah M (2017) Synthesis and characterization of chitosan nanoparticles and their effect on Fusarium head blight and oxidative activity in wheat. Int J Biol Macromol 102:526-538. https://doi.org/10.1016/j.ijbiomac.2017.04.034

Kibar G, Çalıșkan U, Erdem EY, Çetin B (2019) One-pot synthesis of organic-inorganic hybrid polyhedral oligomeric silsesquioxane microparticles in a double-zone temperature controlled microfluidic reactor. J Polym Sci A Polym Chem 57(13):1396-1403. https://doi.org/10.1002/pola.29399

Kim Y, Lee Chung B, Ma M, Mulder WJ, Fayad ZA, Farokhzad OC, Langer R (2012) Mass production and size control of lipidpolymer hybrid nanoparticles through controlled microvortices. Nano Lett 12(7):3587-3591. https://doi.org/10.1021/nl301253v

Lim JM, Swami A, Gilson LM, Chopra S, Choi S, Wu J, Langer R, Karnik R, Farokhzad OC (2014) Ultra-high throughput synthesis of nanoparticles with homogeneous size distribution using a coaxial turbulent jet mixer. ACS Nano 8(6): 6056-6065. https://doi.org/10.1021/nn501371n

Lopes M, Derenne A, Pereira C, Veiga F, Seica R, Sarmento B, Ribeiro A (2016) Impact of the in vitro gastrointestinal passage of biopolymer-based nanoparticles on insulin absorption. RSC Adv 6(24):20155-20165. https://doi.org/10.1039/c5ra26224k

Lu M, Ozcelik A, Grigsby CL, Zhao Y, Guo F, Leong KW, Huang TJ (2016) Microfluidic hydrodynamic focusing for synthesis of nanomaterials. Nano Today 11(6):778-792

Luo G, Du L, Wang Y, Wang KJP (2019) Recent developments in microfluidic device-based preparation, functionalization, and manipulation of nano-and micro-materials

Ma J, Yi C, Li C-W (2020) Facile synthesis and functionalization of color-tunable Ln3+-doped KGdF4 nanoparticles on a microfluidic platform. Mater Sci Eng C Mater Biol Appl 108:110381

Marre S, Jensen KF (2010) Synthesis of micro and nanostructures in microfluidic systems. Chem Soc Rev 39(3):1183-1202. https://doi.org/10.1039/B821324K

Mueller R, Madler L, Pratsinis SE (2003) Nanoparticle synthesis at high production rates by flame spray pyrolysis. Chem Eng Sci 58(10):1969-1976. https://doi.org/10.1016/s0009-2509 (03)00022-8

Mukhopadhyay P, Sarkar K, Chakraborty M, Bhattacharya S, Mishra R, Kundu PP (2013) Oral insulin delivery by selfassembled chitosan nanoparticles: in vitro and in vivo studies in diabetic animal model. Mater Sci Eng C 33(1):376-382. https://doi.org/10.1016/j.msec.2012.09.001

Nitta SK, Numata K (2013) Biopolymer-based nanoparticles for drug/gene delivery and tissue engineering. Int $\mathrm{J}$ Mol Sci 14(1):1629-1654. https://doi.org/10.3390/ijms14011629

No author (2020) Nanomedicine and the COVID-19 vaccines. Nat Nanotechnol 15(12):963-963. https://doi.org/10.1038 /s41565-020-00820-0

Pati F, Adhikari B, Dhara S (2011) Development of chitosantripolyphosphate fibers through $\mathrm{pH}$ dependent ionotropic gelation. Carbohydr Res 346(16):2582-2588. https://doi. org/10.1016/j.carres.2011.08.028

Pessoa ACSN, Sipoli CC, de la Torre LG (2017) Effects of diffusion and mixing pattern on microfluidic-assisted synthesis of chitosan/ATP nanoparticles. Lab Chip 17:2281-2293. https://doi.org/10.1039/C7LC00291B
Prabhakar U, Maeda H, Jain RK, Sevick-Muraca EM, Zamboni W, Farokhzad OC, Barry ST, Gabizon A, Grodzinski P, Blakey DC (2013) Challenges and key considerations of the enhanced permeability and retention effect for nanomedicine drug delivery in oncology. Cancer Res 73(8): 2412-2417. https://doi.org/10.1158/0008-5472.can-12-4561

Pridgen EM, Langer R, Farokhzad OC (2007) Biodegradable, polymeric nanoparticle delivery systems for cancer therapy. Nanomedicine 2(5):669-680. https://doi.org/10.2217 $/ 17435889.2 .5 .669$

Saleh TA (2015) Isotherm, kinetic, and thermodynamic studies on $\mathrm{Hg}$ (II) adsorption from aqueous solution by silica- multiwall carbon nanotubes. Environ Sci Pollut Res Int 22(21):1672116731. https://doi.org/10.1007/s11356-015-4866-z

Saleh T, Gupta V (2012) Characterization of the chemical bonding between $\mathrm{A} 12 \mathrm{O} 3$ and nanotube in MWCNT/A12O3 nanocomposite. Curr Nanosci 8:739-743. https://doi.org/10.2174 /157341312802884418

Schaffazick SR, Guterres SSU, Freitas LD, Pohlmann AR (2003) Physicochemical characterization and stability of the polymeric nanoparticle systems for drug administration. Quim Nova 26(5):726-737. https://doi.org/10.1590/s010040422003000500017

Shchukin DG, Sukhorukov GB (2004) Nanoparticle synthesis in engineered organic nanoscale reactors. Adv Mater 16(8): 671-682. https://doi.org/10.1002/adma.200306466

Skorik YA, Golyshev AA, Kritchenkov AS, Gasilova ER, Poshina DN, Sivaram AJ, Jayakumar R (2017) Development of drug delivery systems for taxanes using ionic gelation of carboxyacyl derivatives of chitosan. Carbohydr Polym 162: 49-55. https://doi.org/10.1016/j.carbpol.2017.01.025

Stoia M, Istratie R, Păcurariu C (2016) Investigation of magnetite nanoparticles stability in air by thermal analysis and FTIR spectroscopy. J Therm Anal Calorim 125(3):1185-1198

Tang Z, Kong N, Zhang X, Liu Y, Hu P, Mou S, Liljeström P, Shi J, Tan W, Kim JS, Cao Y, Langer R, Leong KW, Farokhzad OC, Tao W (2020) A materials-science perspective on tackling COVID-19. Nat Rev Mater 5(11):847-860. https://doi. org/10.1038/s41578-020-00247-y

Volk AA, Epps RW, Abolhasani M (2020) Accelerated development of colloidal nanomaterials enabled by modular microfluidic reactors: toward autonomous robotic experimentation. Adv Mater. https://doi.org/10.1002 /adma.202004495

Yang C-H, Wang L-S, Chen S-Y, Huang M-C, Li Y-H, Lin Y-C, Chen P-F, Shaw J-F, Huang K-S (2016) Microfluidic assisted synthesis of silver nanoparticle-chitosan composite microparticles for antibacterial applications. Pharm Nanotechnol (2):510, 493-500

Zhao C-X, He L, Qiao SZ, Middelberg APJ (2011) Nanoparticle synthesis in microreactors. Chem Eng Sci 66(7):1463-1479. https://doi.org/10.1016/j.ces.2010.08.039

Zhao Y, Liao Z, Xiang Z (2019) Microfluidics for synthesis and morphology control of hierarchical porous covalent organic polymer monolith. Chem Eng Sci 195:801-809

Publisher's note Springer Nature remains neutral with regard to jurisdictional claims in published maps and institutional affiliations. 